Gastroeneterologia. 1944;69:I-IV

\title{
Contents, Vol. 69, 1944
}

\section{INDEX}

An unsere Leser - To our Readers - A nos leeteurs . 1

Bickel, G., Considerations sur le traitement medical de

Гulcère gastro-duodénal ...... 2

Bonnet, G. F., Physio-pathologie de la Cholécystectomie 57,150

Calame, vide Jentzer, A. R.

Demote, M., Les traitements de Гulcère gastro-duodénal par les hormones 31

v. Friedrich, L., Analyse von postoperativen Magenbeschwerden 286

Hemmeler, G., Le traitement de Гulcère gastro-duodénal 188

Jentzer, A. R., et Calame, Les Cholécystites du point de vue chirurgical 177

Lö††ler, H,, Über 14 Todesfälle an Hepatitis epidemica 258

Sjöberg, S.-G., Über die funktionelle Pankreasdiagnostik mit besonderer Berücksichtigung chronischer Pan-kreasaffektionen 233

Wuhrmann, F., und Wunderly $>$, Ch., Über klinische und physikalisch-chemische Untersuchungen des EiweißStoffwechsels beim nephrotischen Symptomenkomplex 121

Wunåerly, Ch., vide Wuhrmann, F. Aus dem Gebiet der Allergie - Problems of Allergy Les problèmes de ГAllergie Lövgren, O., Hypercitricämie bei allergischen Krankheitszuständen. (Vorläufige Mitteilung.) . . . 204 Kleine Mitteilungen - Petites communications Charbonníer, A., Sur un cas d'ileus spasmodique à forme « d'intestin de poulet» 302 